\title{
Ethical, legal, and social issues (ELSI) in rare diseases: a landscape analysis from funders
}

\author{
Adam L. Hartman $\mathbb{( i )}^{1} \cdot$ Anneliene Hechtelt Jonker $\mathbb{( D}^{2} \cdot$ Melissa A. Parisi ${ }^{3} \cdot$ Daria Julkowska $^{4} \cdot$ Nicole Lockhart $^{5}$. \\ Rosario Isasi ${ }^{6}$
}

Received: 16 May 2019 / Revised: 1 August 2019 / Accepted: 29 August 2019 / Published online: 19 September 2019

(C) This is a U.S. government work and not under copyright protection in the U.S.; foreign copyright protection may apply 2019

\begin{abstract}
Recent interest in personalized medicine has highlighted the importance of research in ethical, legal, and social issues (ELSI). Issues in ELSI research may be magnified in the rare diseases population (i.e., small numbers of affected individuals, challenges in maintaining confidentiality, and paucity of treatments for diseases where natural history information may be limited). More than other areas of research, potential barriers include the lack of funding opportunities and appropriate review processes for applications to funding agencies. The ELSI Working Group of the International Rare Diseases Research Consortium (IRDiRC) performed an informal survey on ELSI funding initiatives to learn more about different funding mechanisms and to identify potential gaps in funding opportunities. The Working Group discusses these challenges and highlights the role of funding agencies and partners such as patient advocacy groups, specialists in social sciences and humanities, and clinicians to advance ELSI research in rare diseases.
\end{abstract}

\section{Introduction}

There has been a growing interest worldwide in prospectively assessing the impact and incidental consequences of basic biomedical and applied healthcare

Supplementary information The online version of this article (https:// doi.org/10.1038/s41431-019-0513-3) contains supplementary material, which is available to authorized users.

Adam L. Hartman

adam.hartman@nih.gov

1 Division of Clinical Research, National Institute of Neurological Disorders and Stroke, NIH, Rockville, MD 20852, USA

2 IRDiRC Scientific Secretariat, Inserm US 14, 75014 Paris, France

3 Intellectual \& Developmental Disabilities Branch, Eunice Kennedy Shriver National Institute of Child Health and Human Development, NIH, Bethesda, MD 20892, USA

4 European Joint Programme on Rare Diseases, Institut Thématique Génétique, Génomique et Bioinformatique, INSERM, 75013 Paris, France

5 Division of Genomics and Society, National Human Genome Research Institute, NIH, Bethesda, MD 20892, USA

6 Institute for Bioethics and Health Policy, Department of Human Genetics, Leonard M. Miller School of Medicine, University of Miami, Miami, FL 33136, USA research on individuals, families and societies as a whole. A multidisciplinary field of study, collectively known as ethical, legal and social issues (ELSI), has seen considerable growth over the last three decades, particularly in the USA, due in large part to the launch of the National Human Genome Research Institute's (NHGRI) ELSI Research Program, which was set up as an integral part of the Human Genome Project (HGP) [1-3]. Similar efforts have followed in other regions, such as in Canada with the introduction of the GE3LS Program (genomics, ethical environmental, economic, legal and social aspects) and in Europe, where the Horizon 2020 Research \& Innovation Framework Program integrates ELSI in each project financed by the European Commission in addition to the dedicated funding opportunities fostering ethics and innovation. These efforts, while successful in many respects as demonstrated by their impact in policy development (e.g., the Genetic Nondiscrimination Act of 2008 in the U.S. and revisions to the U.S. Common Rule $[4,5])$, have yet to realize their full potential for a wide range of stakeholders (e.g., policymakers, scientists, regulatory and funding agencies) but particularly for people affected by genetic diseases. Indeed, rare disease research has only recently received increased attention as awareness of the unique and sensitive ELSI issues for these conditions has also intensified [2, 6-11]. 


\section{ELSI challenges in rare disease research}

Growing understanding of the need to prospectively identify, analyze, and manage actual or emerging ELSI issues and challenges has spurred greater attention to integrating ELSI studies in genetics research and medicine. The latter has prompted a paradigm shift fostering greater appreciation of the unique, common-yet nuanced-and complex ELSIrelated issues that arise in the rare disease field. In fact, conventional ethical issues such as autonomy (e.g., consent), privacy, and governmental oversight considerations present in research on common diseases are further amplified in the rare disease area because of the small numbers of study participants, the difficulty in maintaining confidentiality for those affected individuals because of the rarity of their conditions, and the paucity of treatments available for conditions for which natural history information is often sparse. This mandates a balanced approach in the application of the ethical principles of autonomy, solidarity, beneficence, justice, and reciprocity, to cite just a few [12-14]. At the same time, the expectations, concerns, attitudes, and priorities of the rare disease community do not overlap entirely with those present in other vulnerable or underrepresented populations (one example is allocation of healthcare expenditures for common diseases in underrepresented populations vs. rare diseases in all populations), thus requiring more thoughtful consideration [7].

There are many reasons for these challenges. Patients and caregivers living with a rare disease appear to face inequitable hurdles. A long diagnostic odyssey, limited access to treatments and related social services support, and life plans change as inevitable consequence of diagnosis (and treatments when available), paired with a heavy financial impact are far more common in rare diseases, compared with more common diseases $[6,15,16]$. Because many rare diseases involve genetic variants that are expressed at a low frequency in the general population, some additional ELSIrelated issues might be highlighted. Examples include the application of gene modification technologies (e.g., gene editing) and the integration of stem cell research (e.g., derivation of pluripotent stem cell lines) to deliver precision therapies. The importance of ELSI in this context was recently highlighted by the call for a moratorium on heritable genome editing of the germline [17]. In addition, asymmetrical opportunities for participation in research, due to fewer funding initiatives, size and population characteristics, and blurring lines between research and clinical care further exacerbate concerns over the adequacy of participants' safeguards, including those directed to address or to mitigate therapeutic misconception (or overestimation), discrimination, and stigmatization.

The scarcity of resources, in terms of patient/participant population, access to clinical data and biospecimen samples, and distinct data needs (e.g., photography to enhance phenotypic comparisons, etc.), highlights the ethical and scientific imperative of data sharing-beyond jurisdictional borders-which is of the utmost importance for rare diseases research. At the same time, it brings to the forefront considerations of privacy, confidentiality, and security that must take into account the interests and perspectives of those patients/participants and their families, together with those of investigators and regulators alike [13, 18-20]. For example, patients with rare diseases are often individually identifiable, particularly if their quest for services or treatments brings them into the public sphere. In addition, access to potentially life-saving therapies may require compassionate use and increased risk to the research participant because a randomized clinical trial may not be feasible for a rare disorder.

Furthermore, divergence in policy frameworks (laws, regulations, professional guidelines) for rare diseases and heterogeneity in sociocultural views across countries further challenges a field that often uses novel and disruptive technologies to overcome the hurdles caused by the limited access to widely available treatments, as with more common diseases. Different legal incentives in different countries that stimulate the development of orphan drugs [21-23] for rare disorders further exacerbate the ethical and social debate, particularly in terms of global access to safe and efficient treatments [24, 25].

Fortunately, rare diseases research is characterized by the incredible drive and influence of patient organizations, who participate in and lead, fund, and direct the research agenda [26-28]. Specifically, patient advocacy organizations are increasingly involved in trial design (some by mandate of the funding agency) and may play a key role in recruitment for studies involving human participants and as part of research governance structures (e.g., the US Patient Centered Outcomes Research Institute, PCORI). These organizations have a high level of social engagement and visibility that extends beyond the research context, exerting political influence by impacting and shaping public policy and legislation.

At the organizational level, funding agencies have encountered difficulties in fully integrating ELSI proposals into their existing funding frameworks. For instance, the multidisciplinary and unique nature of ELSI studies (including both rare and more common diseases) might not fit well within the mission of biomedical funding agencies with a narrow focus or a limited budget. Even for larger biomedical agencies with greater resources, challenges arise in their limited expertize or capacity to fairly review and scientifically oversee ELSI applications; the situation is magnified for rare diseases because of the characteristics noted previously. In addition, these agencies and smaller private foundations are faced with responding to 
stakeholders, who consider the convening of diverse multidisciplinary groups, with seemingly disparate interests, to be outside of their scope or capabilities. Furthermore, some investigators in the social sciences and humanities remain unfamiliar with funding opportunities available to bridge rare diseases with their respective fields (law, sociology, philosophy, etc.) thus further hindering work in this area. Finally, social sciences and humanities researchers may not be particularly aware of the specific ELSI-related issues facing people with rare diseases, and of those encountered during the clinical translation process. These issues might very well be considered for such researchers as fertile ground for future studies that could be translated into tangible benefits to those affected by rare diseases. There has been some success in addressing these challenges (e.g., newborn screening) but challenges remain in other aspects of ELSI research.

\section{The current landscape}

To identify gaps in funding opportunities and gain a broader understanding of the ELSI research landscape in rare diseases, the International Rare Diseases Research Consortium (IRDiRC) Funders Constituent Committee [29, 30] set up an informal survey on ELSI funding initiatives (Supplementary Material). IRDiRC is a consortium of public and private research funding bodies and patient organizations committed to rare diseases research. This survey included 30 public funding members located in Australia, Asia, Europe, and North America. IRDiRC members each spend at least \$2million (USD) per year on rare diseases research. While this effort was based on a self-reported survey, it addressed the following issues: (i) an overview of current and past funding calls and announcements related to ELSI connected to rare diseases (Table 1), (ii) identification of ELSI priority issues in the said funding opportunities; and, (iii) identification of other potential gaps (and ideas for future funding calls) in ELSI-related research in rare diseases (Table 2).

Three main observations arise from these findings. Notably, funding opportunities for rare diseases addressing ELSI-related issues were the exception. Overall, some funding announcements integrated ELSI research as part of a more general opportunity available to researchers including those who focus on rare diseases, while in other calls funders had established ELSI programs integrating rare diseases initiatives in themselves (Table 1). One example of the few ELSI-rare disease-specific funding opportunities is entitled, "Human and Social Sciences \& Rare Diseases" from the French Foundation for Rare Diseases (FFRD) [31]. This opportunity is specifically designed to better understand the social, familial, and individual consequences to rare diseases by investigating the knowledge about the specific impact of a rare disease in terms of disability and quality of life. The funding announcement ultimately aims to improve the life course of the person suffering from a rare disease and his or her caregivers, throughout the process of searching for a diagnosis until treatment, by bringing together researchers in social and human sciences, experts in medico-social support and rare diseases patient organizations. Over the last 5 years, FFRD funded 34 projects in France involving 116 research teams and 50 patients' organizations, working on various rare diseases, and social/ethical aspects ranging from psychosocial consequences of rare diseases to social adaptation of adolescents affected by rare diseases. The success of this funding call has been attributed to the regional managers who actively promoted the initiative to constituencies from disparate backgrounds (e.g., clinicians, ELSI researchers, and patient advisory groups) (R. Favresse, D. Desir-Parseille, personal communication, July 29, 2019). In addition to the importance of this work, these data suggest that there are investigators capable of carrying out this type of work, which is one challenge faced by biomedical funding agencies looking to support work in the social sciences. On the other hand, the experience of FFRD showed that gathering of necessary expertize to form relevant consortia is challenging; while clinicians and patients collaborate regularly, experts in social sciences and humanities are difficult to find because they are not "natural" partners in rare diseases research.

In some funding environments, rare disease investigators apply to an ELSI-related funding opportunity that might be broader in its ELSI scope. As an example, several funders in North America, Japan and Europe have calls for ELSI as part of genomics research project, such as the U.S. National Institutes of Health (NIH) "ELSI of Genomics Research Project Grant Program", the Japanese "Leading the way to Genomic Medicine by ELSI Research Program" or the German "Ethical, Legal and Social Aspects of the Modern Life Sciences" [32-34].

While the number of funding opportunities with an ELSI component is similar in North America, Europe, and to some degree Japan, most European projects appear to have one component of each general funding opportunity systematically dedicated to ELSI topics, whereas North American ELSI funding calls (USA, Canada) appear to encourage study of more specific ELSI-related topics. An example of general funding opportunities with an ELSI component are the EU research and Innovation program Horizon 2020 (H2020), which have a specific section "Science with and for Society" that is dedicated to societal and ethics aspects of research and innovation [35]. An example of a funding opportunity with an ELSI component on a more specific topic is the funding announcement from 


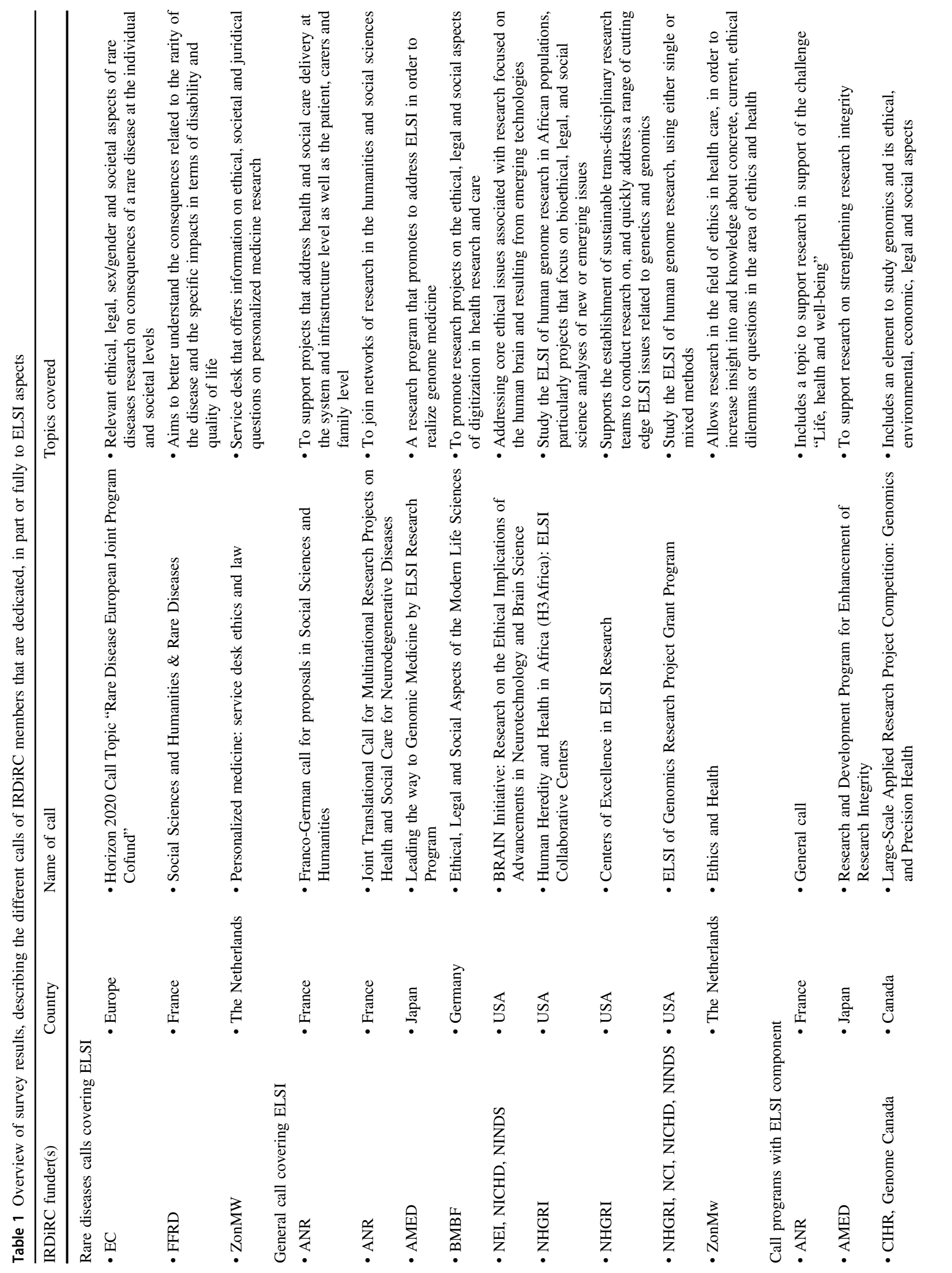




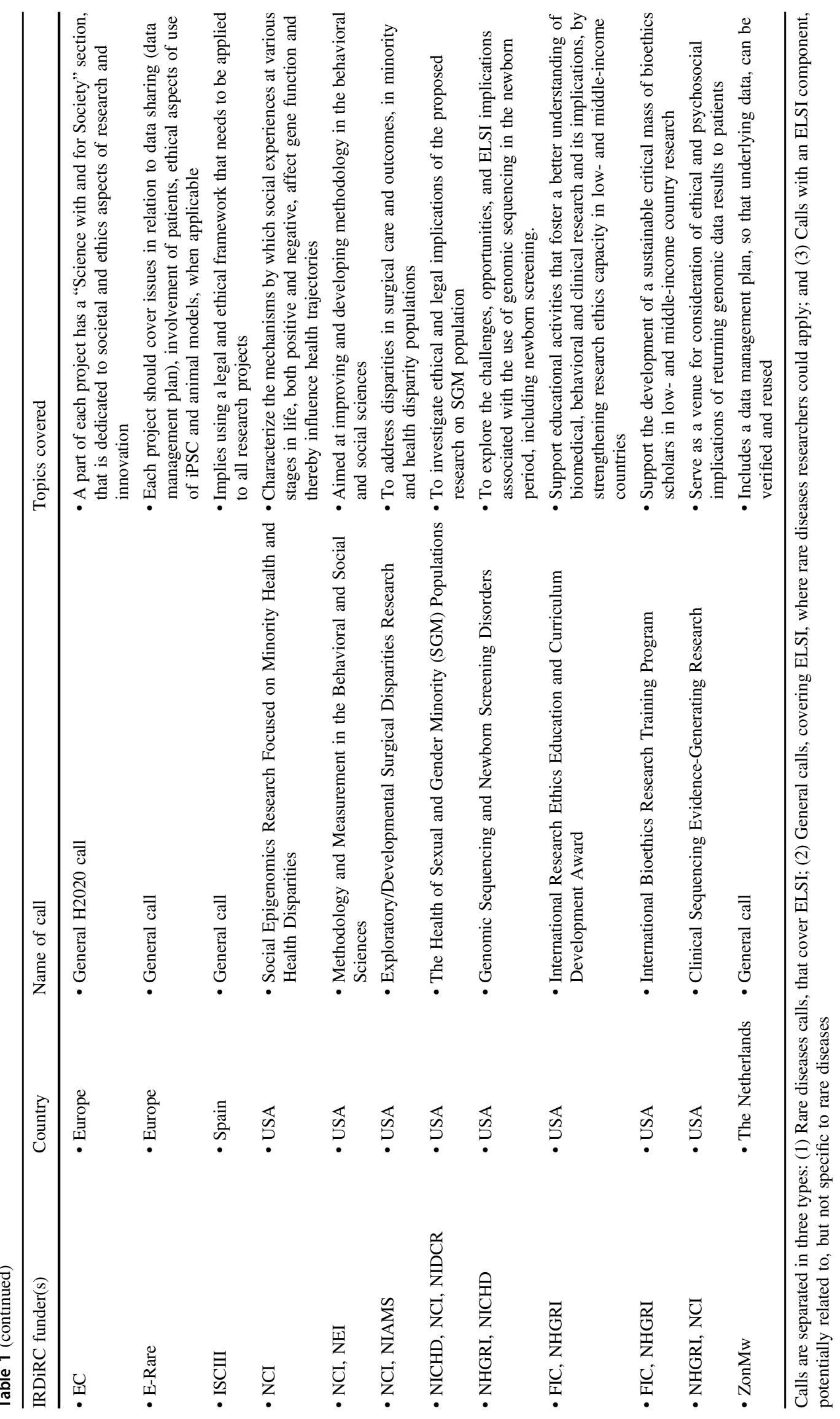


Table 2 Topics that should be covered by ELSI rare diseases funding calls, as indicated by IRDiRC funders. The topics are subdivided in categories, but many of the topics interact with different categories

\begin{tabular}{|c|c|}
\hline $\begin{array}{l}\text { Topics for ELSI rare diseases } \\
\text { funding calls }\end{array}$ & Category \\
\hline Data collection & $\begin{array}{l}\text { - Privacy regulations (GPDR) and impact data collection for rare } \\
\text { disease clinical studies } \\
\text { - Integration of multiple sources of data } \\
\text { - Data management plans } \\
\text { - Access to rights }\end{array}$ \\
\hline Data sharing & $\begin{array}{l}\text { - Privacy } \\
\text { - Equity of access } \\
\text { - Quality of information and how it is disseminated } \\
\text { - Best practices for cross-border data sharing compliant with ethics } \\
\text { requirements and data protection laws }\end{array}$ \\
\hline $\begin{array}{l}\text { Challenges of living with a rare } \\
\text { disease }\end{array}$ & $\begin{array}{l}\text { - Economic analysis on burden of rare diseases } \\
\text { - Compensation strategies put in place by the person with a RD and/or } \\
\text { relative } \\
\text { - Evaluation of the direct/indirect costs of living with a RD } \\
\text { - Social life and citizenship } \\
\text { - Decision making of people affected with RD } \\
\text { - Health literacy } \\
\text { - Knowledge/perception of disability by third party } \\
\text { - Impact of a RD on siblings or the family } \\
\text { - Impact of rarity of disease on life plan (including parenthood) } \\
\text { - Assessment of specific situations of disruption in the health follow-up } \\
\text { - Access to care }\end{array}$ \\
\hline Consent and patient information & $\begin{array}{l}\text { - Disclosure of information to family members } \\
\text { - Informed consent } \\
\text { - Protection of patient information } \\
\text { - Protection of people in relation to specific vulnerabilities }\end{array}$ \\
\hline Patient engagement & $\begin{array}{l}\text { - Patient inclusion in design of research programs and their evaluation } \\
\text { - Role of Patient Organizations in supporting families, supporting } \\
\text { social participation } \\
\text { - Collaboration of different stakeholders in health/social/medico- } \\
\text { social fields } \\
\text { - Access to learning }\end{array}$ \\
\hline
\end{tabular}

multiple National Institute of Health (NIH) institutes entitled "The Health of Sexual and Gender Minority (SGM) Populations," in which a part of the call is dedicated to investigate ethical and legal implications of the proposed research on SGM population [36]. In the US, ELSI research funding opportunities have tended to relate to more general areas of biological sciences or medicine which could include rare diseases. For example, several NIH Institutes and Centers have participated in ongoing funding opportunities for ELSI research related to genetic and genomic research. From fiscal year 2015-2018, these funding opportunities related to genomics funded 31 awards. In fiscal years 2017 and 2018, several NIH Institutes and Centers collaborated on funding opportunities related to the ethical implications of advancements in neurotechnology with a total of nine applications funded. Finally, in 2017 and 2018 the NIH Human Heredity and Health in Africa program issued a funding opportunity for ELSI research related to human genome research in African populations funded five applications. Unfortunately, specific reasons underlying the success of individual applications cannot be disclosed but the data suggest that there is a fundable group of investigator teams from disparate backgrounds (and in contrast to the FFRD experience, there was no active systematic prospective engagement with individuals from different professional backgrounds; unfortunately, because of other structural differences between funding infrastructures in the USA and France, a direct comparison of the two programs would not be valid). Overall, funding opportunities with an ELSI component cover personalized medicine, genomics and gene discovery, public health programs such as newborn screening, social sciences, and ethics [37]. Varying approaches have been used in individual jurisdictions, largely for either historical reasons or statutory differences in oversight of scientific research budgets (e.g., executive branch, legislative branch, or some combination of the two). Private foundations might target specific opportunities based on their specific interests, as well.

\section{A proposal for moving forward}

Survey respondents identified several perceived gaps and challenges in the current funding opportunity and research 
landscape (Table 2). Some of these topics involve ELSI topics for both common and rare diseases. However, some are more critical for rare disease. Based on the challenges noted here, a blueprint for advancing ELSI research in rare diseases and for integrating ELSI analysis in rare diseases research in general becomes more apparent. As a first step, a more comprehensive assessment of the current funding landscape is needed. Our informal survey only included large funding agencies and did not include some continents (e.g., South America and Africa), nor did it go into detail of individual countries or regional/state funding programs. There might be ELSI-related research questions that are unique to some of these regions. Thus, one could consider that the survey underestimated the prevalence of this type of funding opportunity. On the other hand, larger funding agencies are more likely to have the programmatic and financial bandwidth to support and identify this type of work. Another issue identified is the diversity of national health care systems and subsequent complexity of transposition of specific ELSI research results to broader context (covering multiple countries). Finally, as indicated by the experience of the FFRD, the success of the studies is highly dependent on the engagement by regional directors of relevant stakeholders including specialists in social sciences and humanities, clinicians, and patients because such collaboration and respective working methodology is not yet considered as "natural" and requires important efforts of engaged parties.

Notwithstanding these challenges, the survey identified potential items that could be included in future funding initiatives. Examples included data collection, data sharing, challenges of living with a rare disease, consent and patient information, and patient engagement. Input from patients, caregivers, and their advocacy groups (amongst other stakeholders) would help prioritize which ELSI issues deserve attention and funding support to address them. These stakeholders also could convene investigators in the social sciences and humanities in a forum with clinicians and funding agencies to facilitate community building, and encourage their participation in a broader research agenda for ELSI-related issues in rare diseases.

\section{Discussion}

The IRDiRC informal survey was the first step in identifying some of the gaps in the current funding landscape for ELSI issues in rare diseases. A dedicated, strategic workshop, organized by E-Rare [38] in collaboration with IRDiRC ELSI working group is now in preparation and is expected to lead to further identification of most important challenges in this area. NIH released a funding announcement [39] to supplement a variety of existing grants (including rare diseases research) to conduct bioethics research. The tasks of identifying, analyzing, and managing ELSI issues and challenges arising in rare diseases should be considered as essential pillars of innovation in this area. Most important is understanding and meeting the expectations, concerns, attitudes, and priorities of the rare disease community. Setting and re-appraising the ELSI research agenda is thus a fundamental step toward those goals.

Acknowledgements Members and contributors of the IRDiRC ELSI Working group included ALH, HD, RI, DJ, NL and MP. In addition, we would like to thank Domenica Taruscio (ISS, Italy) and Manuel Posada (ISCIII, Spain) for their review and feedback on the manuscript.

Funding The IRDiRC Scientific Secretariat was supported by the European FP7 contract "SUPPORT-IRDiRC"( ${ }^{\circ}$ 305207). Daria Julkowska received funding from the European Union's Horizon 2020 Research and Innovation Program under grant agreement EJP RD N ${ }^{\circ}$ 825575 .

\section{Compliance with ethical standards}

Conflict of interest The authors declare no competing interests. The content is solely the responsibility of the authors and does not necessarily represent the official views of the National Institute of Neurological Disorders and Stroke, Eunice Kennedy Shriver National Institute of Child Health and Human Development, National Human Genome Research Institute, National Institutes of Health, Department of Health and Human Services, or the US Government.

Publisher's note Springer Nature remains neutral with regard to jurisdictional claims in published maps and institutional affiliations.

\section{References}

1. National Research Council. Mapping and sequencing the human genome. Washington, DC: The National Academies Press; 1988. https://www.nap.edu/read/1097/chapter/1. Accessed 13 Jan 2019.

2. McEwen JE, Boyer JT, Sun KY, Rothenberg KH, Lockhart NC, Guyer MS. The Ethical, Legal, and Social Implications Program of the National Human Genome Research Institute: reflections on an ongoing experiment. Annu Rev Genomics Hum Genet. 2014;15:481-505.

3. Meslin EM, Thomson EJ, Boyer JT. The Ethical, Legal, and Social Implications Research Program at the National Human Genome Research Institute. Kennedy Inst Ethics J. 1997;7:291-8.

4. US Government Publishing Office. Genetic Information Nondiscrimination Act of 2008. 2008. https://www.govinfo.gov/ content/pkg/PLAW-110publ233/pdf/PLAW-110publ233.pdf. Accessed 27 Jan 2019.

5. US Government Publishing Office. Revised Common Rule. 2017. https://www.govinfo.gov/content/pkg/FR-2017-01-19/pdf/201701058.pdf. Accessed 27 Jan 2019.

6. Gainotti S, Mascalzoni D, Bros-Facer V, Petrini C, Floridia G, Roos $\mathrm{M}$, et al. Meeting patients' right to the correct diagnosis: ongoing international initiatives on undiagnosed rare diseases and ethical and social issues. Int J Environ Res Public Health. 2018;15:10.

7. McCormack P, Kole A, Gainotti S, Mascalzoni D, Molster C, Lochmüller H, et al. 'You should at least ask'. The expectations, hopes and fears of rare disease patients on large-scale data and 
biomaterial sharing for genomics research. Eur $\mathrm{J}$ Hum Genet. 2016;24:1403-8.

8. Bereza E. The use of clinical databases in disease outcomes research: Is the ethics of IRB review keeping up? Mult Scler. 2017;23:1325-7.

9. Polisena J, Burgess M, Mitton C, Lynd LD. Engaging the Canadian public on reimbursement decision-making for drugs for rare diseases: a national online survey. BMC Health Serv Res. 2017;17:372.

10. Hallowell N, Parker M, Nellåker C. Big data phenotyping in rare diseases: some ethical issues. Genet Med. 2019;21:272-4.

11. Parker LS, Sankar PL, Boyer J, Jean McEwen JD, Kaufman D. Normative and conceptual ELSI research: what it is, and why it's important. Genet Med J Am Coll Med Genet. 2019;21:505-9.

12. Knoppers BM. Framework for responsible sharing of genomic and health-related data. HUGO J. 2014;8:3.

13. Mascalzoni D, Dove ES, Rubinstein Y, Dawkins HJ, Kole A, McCormack $\mathrm{P}$, et al. International charter of principles for sharing bio-specimens and data. Eur J Hum Genet. 2015;23:721-8.

14. Gainotti S, Turner C, Woods S, Kole A, McCormack P, Lochmuller $\mathrm{H}$, et al. Improving the informed consent process in international collaborative rare disease research: effective consent for effective research. Eur J Hum Genet. 2016;24:1248-54.

15. Schieppati A, Henter J-I, Daina E, Aperia A. Why rare diseases are an important medical and social issue. Lancet. 2008;371:2039-41.

16. Cannizzo S, Lorenzoni V, Palla I, Pirri S, Trieste L, Triulzi L, et al. Rare diseases under different levels of economic analysis: current activities, challenges and perspectives. RMD Open. 2018;4:e00794.

17. Lander ES, Baylis F, Zhang F, Charpentier E, Berg P, Bourgain C, et al. Adopt a moratorium on heritable genome editing. Nature. 2019;567:165.

18. Baker D, Knoppers BM, Phillips M, van Enckevort D, Kaufmann $\mathrm{P}$, Lochmuller $\mathrm{H}$, et al. Privacy-preserving linkage of genomic and clinical data sets. IEEE/ACM Trans Comput Biol Bioinform. 2019;16:1342-8.

19. Kodra Y, Weinbach J, Posada-de-la-Paz M, Coi A, Lemonnier SL, van Enckevort D, et al. Recommendations for improving the quality of rare disease registries. Int J Environ Res Public Health. 2018;15. https://doi.org/10.3390/ijerph15081644.

20. Monaco L, Crimi M, Wang CM. The challenge for a European network of biobanks for rare diseases taken up by RD-Connect. Pathobiol J Immunopathol Mol Cell Biol. 2014;81:231-6.

21. FDA. FDA: Orphan Drug Act. 1983. https://www.fda.gov/ ForConsumers/ConsumerUpdates/ucm2005643.htm. Accessed 27 Jan 2019.

22. European Commission. EC: Regulation No 141/2000 of the European Parliament and of the Council on Orphan Medical Products. 2000. https://ec.europa.eu/health/sites/health/files/files/ eudralex/vol-1/reg_2000_141_cons-2009-07/reg_2000_141_ cons-2009-07_en.pdf. Accessed 27 Jan2019.

23. PMDA. PMDA: The Article 77-2 of the Pharmaceutical Affairs Law. 1985. https://www.mhlw.go.jp/english/policy/health-medica 1/pharmaceuticals/d1/05.pdf. Accessed 27 Jan 2019.
24. Gericke CA, Riesberg A, Busse R. Ethical issues in funding orphan drug research and development. $\mathrm{J}$ Med Ethics. 2005;31:164-8.

25. Rodriguez-Monguio R, Spargo T, Seoane-Vazquez E. Ethical imperatives of timely access to orphan drugs: is possible to reconcile economic incentives and patients' health needs? Orphanet J Rare Dis. 2017;12:1.

26. Heeney C, Hawkins $\mathrm{N}$, de Vries J, Boddington P, Kaye J. Assessing the privacy risks of data sharing in genomics. Public Health Genomics. 2011;14:17-25.

27. Hardavella G, Bjerg A, Saad N, Jacinto T, Powell P. How to optimise patient and public involvement in your research. Breathe. 2015;11:223-7.

28. PARADIGM. PARADIGM, patients active in research and dialogues for an improved generation of medicines. 2019. https://imiparadigm.eu. Accessed 27 Jan 2019.

29. IRDiRC. International Rare Diseases Research Consortium. IRDiRC. 2019. http://www.irdirc.org/. Accessed 13 Jan 2019.

30. Dawkins HJS, Draghia-Akli R, Lasko P, Lau LPL, Jonker AH, Cutillo CM, et al. Progress in rare diseases research 2010-6: an IRDiRC perspective. Clin Transl Sci. 2018;11:11-20.

31. FFRD. Appel à projets de recherche 2018 «Sciences humaines et sociales \& maladies rares». https://www.fondation-Mal. Contentuploads2018022018SHSMR.pdf. Accessed 13 Jan 2019.

32. NIH. PA-17-325: Ethical, Legal, and Social Implications (ELSI) of Genomics Research Project Grant Program (R01). 2017. https:// grants.nih.gov/grants/guide/pa-files/pa-17-325.html. Accessed 28 Jan 2019

33. Japan Agency for Medical Research and Development. Awarded Projects under the Research and Development Program for Enhancement of Research Integrity. 2016. https://www.amed. go.jp/en/news/program/1001C_00010.html. Accessed 28 Jan 2019.

34. BMBF-Internetredaktion. Bekanntmachung - BMBF. Bundesminist. Für Bild. Forsch. - BMBF. 2018. https://www.bmbf.de/ foerderungen/bekanntmachung-2103.html. Accessed 28 Jan 2019.

35. Horizon 2020-European Commission. Science with and for Society. Horiz. 2020 - Eur. Comm. 2013. https://ec.europa.eu/ programmes/horizon2020/en/h2020-section/science-and-society. Accessed 28 Jan 2019.

36. NIH. The Health of Sexual and Gender Minority (SGM) Populations (R21 Clinical Trial Optional). 2013. https://grants.nih.gov/ grants/guide/pa-files/PA-18-040.html. Accessed 28 Jan 2019.

37. Berg JS, Agrawal PB, Bailey DB, Beggs AH, Brenner SE, Brower $\mathrm{AM}$, et al. Newborn sequencing in genomic medicine and public health. Pediatrics. 2017;139. https://doi.org/10.1542/peds.20162252.

38. E-Rare. ERA-net for research programmes on rare diseases. 2018. http://www.erare.eu. Accessed 16 Feb 2019.

39. NIH Administrative Supplement for Research on Bioethical Issues (Admin Supp Clinical Trial Optional) - PA-19-217. 2019. https:// grants.nih.gov/grants/guide/pa-Files/PA-19-217.html. 